\title{
Acute effects of meal fatty acid composition on insulin sensitivity in healthy post-menopausal women
}

\author{
M. D. Robertson ${ }^{1}$, K. G. Jackson ${ }^{2}$, B. A. Fielding ${ }^{1}$, C. M. Williams ${ }^{2}$ and K. N. Frayn ${ }^{1}$ \\ ${ }^{1}$ Oxford Centre for Diabetes, Endocrinology and Metabolism, University of Oxford, Oxford OX2 6HE, UK \\ ${ }^{2}$ Hugh Sinclair Unit for Human Nutrition, School of Food Biosciences, University of Reading, Reading RG6 6AP, UK
}

(Received 27 March 2002 - Revised 11 July 2002 - Accepted 11 August 2002)

\begin{abstract}
Postprandial plasma insulin concentrations after a single high-fat meal may be modified by the presence of specific fatty acids although the effects of sequential meal ingestion are unknown. The aim of the present study was to examine the effects of altering the fatty acid composition in a single mixed fat-carbohydrate meal on glucose metabolism and insulin sensitivity of a second meal eaten $5 \mathrm{~h}$ later. Insulin sensitivity was assessed using a minimal model approach. Ten healthy post-menopausal women underwent four two-meal studies in random order. A high-fat breakfast ( $40 \mathrm{~g}$ fat) where the fatty acid composition was predominantly saturated fatty acids (SFA), $n-6$ polyunsaturated fatty acids (PUFA), long-chain $n-3$ PUFA or monounsaturated fatty acids (MUFA) was followed $5 \mathrm{~h}$ later by a low-fat, high-carbohydrate lunch $(5.7 \mathrm{~g}$ fat), which was identical in all four studies. The plasma insulin response was significantly higher following the SFA meal than the other meals after both breakfast and lunch $(P<0.006)$ although there was no effect of breakfast fatty acid composition on plasma glucose concentrations. Postprandial insulin sensitivity $\left(\mathrm{S}_{\mathrm{I}(\mathrm{Oral})}\right)$ was assessed for $180 \mathrm{~min}$ after each meal. $\mathrm{S}_{\mathrm{I}(\text { Oral })}$ was significantly lower after lunch than after breakfast for all four test meals $(P=0.019)$ following the same rank order (SFA $<n$-6 PUFA $<n$-3 PUFA $<$ MUFA) for each meal. The present study demonstrates that a single meal rich in SFA reduces postprandial insulin sensitivity with 'carry-over' effects for the next meal.
\end{abstract}

Fatty acids: Postprandial metabolism: Insulin sensitivity: Post-menopausal women

Insulin insensitivity has been postulated to be the underlying factor linking type 2 diabetes, hypertension and cardiovascular disease (Reaven, 1988). Epidemiological evidence now suggests that a high intake of dietary fat is associated with impaired insulin sensitivity $\left(\mathrm{S}_{\mathrm{I}}\right)$, which may be modulated by the type of fatty acids in the diet (Vessby, 2000). It has also been shown that postprandial plasma insulin concentrations after a single meal may be modified by the presence of specific fatty acids. However, results have been conflicting. Rasmussen et al. (1996) showed that insulin release was stimulated by butter but not by olive oil (rich in monounsaturated fatty acids; MUFA) in patients with type 2 diabetes, whereas in healthy subjects, mixed meals containing MUFA have given higher postprandial plasma insulin and glucose concentrations compared with meals rich in polyunsaturated fatty acids (PUFA) (Joannic et al. 1997; Pedersen et al. 1999). Other studies, however, have found no effect of meal fatty acid composition on postprandial insulin or glucose levels when given as part of a mixed meal either in the morning (Thomsen et al. 1999) or evening (Zampelas et al. 1994). We have emphasized the need to study the effects of sequential meals on postprandial metabolism since this reflects the more usual metabolic state in Westernized societies (Fielding et al. 1996; Evans et al. 1998). It has already been demonstrated that following sequential meal ingestion, chylomicrons are released rapidly into the circulation following the second meal. Ercan et al. (1994) have used a sequential meal protocol to show that plasma glucose and insulin areas under the curve (AUC) were higher in response to a second meal irrespective of the fat content of the first meal. These findings are in contrast with those of Frape et al. (1997a,b, 1998), who demonstrated clearly that lunch-time glucose tolerance was impaired by a fatty breakfast. However, neither of these studies addressed the potential influence of breakfast

\footnotetext{
Abbreviations: AUC, area under the curve; GE, glucose effectiveness; MUFA, monounsaturated fatty acids; NEFA, non-esterified fatty acids; PUFA, polyunsaturated fatty acids; SFA, saturated fatty acids; $S_{I}$, insulin sensitivity.

*Corresponding author: Dr M. D. Robertson, fax +44 1865 224652, email denise.robertson@oxlip.ox.ac.uk
} 
fatty acid composition, which may be important as part of the 'second-meal' effect.

The aim of the present study was to examine whether altering the fatty acid composition in a single mixed fatcarbohydrate meal had 'carry-over' effects on glucose metabolism and $\mathrm{S}_{\mathrm{I}}$ assessed using the minimal model approach (Caumo et al. 2000) for a second meal eaten $5 \mathrm{~h}$ later. Other data from the present study on aspects of lipid metabolism have been published previously (Jackson et al. 2002; Robertson et al. 2002a).

\section{Subjects and methods}

\section{Subjects}

Ten healthy post-menopausal women (mean age 56 (range 50-63) years; mean BMI $25 \cdot 0$ (range 20.6-32.0) $\mathrm{kg} / \mathrm{m}^{2}$ ) were studied on four occasions. All subjects satisfied the following inclusion criteria: they were weight-stable and were not currently taking fatty acid supplements or medication likely to affect either gastrointestinal motility or lipid metabolism (including hormone replacement therapy). They had no previous history of hyperlipidaemia, gastrointestinal or endocrine disease. Subjects were recruited following screening for fasting blood lipid and glucose levels, which were all within normal limits (triacylglycerol, mean 1.2 (range $0.7-1.5$ ) $\mathrm{mmol} / \mathrm{l}$; total cholesterol, mean 5.7 (range 4.4-6.5) mmol/l; glucose, mean $5 \cdot 1$ (range $4 \cdot 5-5 \cdot 8$ ) mmol/l). Written informed consent was obtained from all subjects and the study was approved by the University of Reading and Central Oxford Research Ethics Committees.

\section{Study design}

The experiment was set up as a single-blind, randomized study with four separate postprandial study days occurring at least 1 month apart. Two carbohydrate-rich meals were provided $5 \mathrm{~h}$ apart, the first meal high in fat $(41 \mathrm{~g})$ and the second meal low in fat $(6 \mathrm{~g})$. The fatty acid composition of the high-fat first meal was varied on each visit. Postprandial $S_{I}$ following each meal was assessed using a minimal model approach (Caumo et al. 2000).

\section{Study-day procedures}

All studies were performed following a standardized lowfat evening meal $(<10 \mathrm{~g}$ fat) and an overnight fast. In the morning, an indwelling intravenous cannula was inserted into an antecubital vein under local anaesthetic (1\% lignocaine). At time zero, subjects were given one of four test meals (Table 1) consisting of Rice Krispies, banana and a drink containing $40 \mathrm{~g}$ test oil (Table 2). The test oils were chosen to provide meals enriched with either saturated fatty acids (SFA; palm oil), n-6 PUFA (safflower-seed oil; Anglia Oils Ltd, Kingson upon Hull, UK), long-chain $n$-3 PUFA (EPAX 3000TG; Pronova Biocare, Aaslund, Norway. The EPAX 3000TG was supplied under $\mathrm{N}_{2}$ with a new bottle used for each study to minimize oxidation) or MUFA (olive oil; Tesco, Cheshunt, Herts., UK). The EPAX 3000TG fish oil was diluted (1:1) with safflower-seed oil in order to reduce the proportion of SFA in the $n$-3 PUFA test meal and to improve the palatability of the dietary fish oil. Fasting and postprandial blood samples were taken at regular intervals for $300 \mathrm{~min}$. At $300 \mathrm{~min}$, a second meal (low-fat, high-carbohydrate) was consumed by the subjects. This meal was identical on all four visits. Blood samples were taken at 10, 20, 30, 60, 120 and $180 \mathrm{~min}$ after the second meal. Between meals subjects did not consume any other food other than water, which was provided ad libitum. The test meals were well tolerated by all subjects.

\section{Blood analyses}

Whole blood for metabolite and insulin determination was collected into heparinized syringes (Sarstedt, Leicester, Leics., UK and L.I.P., Shipley, W. Yorks, UK). Plasma glucose (Instrumentation Laboratory, Warrington, Ches., UK) and non-esterified fatty acid (NEFA) concentrations (Alpha Laboratories, Eastleigh, Hants, UK) were measured enzymically using an Instrumentation Laboratory Monarch automated analyser. Metabolites were batch-analysed and exhibited an intra-assay variation of less than $2.5 \%$. Insulin was measured by radioimmunoassay using a commercially available kit (Pharmacia \& Upjohn, Milton Keynes,

Table 1. Macronutrient composition of test meals*

\begin{tabular}{|c|c|c|c|c|}
\hline & Carbohydrate (g) & Fat $(\mathrm{g})$ & Protein $(\mathrm{g})$ & Energy (kJ) \\
\hline \multicolumn{5}{|l|}{ Breakfast } \\
\hline Test oil $(40 \mathrm{~g})$ & 0 & 40 & 0 & 1478 \\
\hline Skimmed milk (250 g) & $12 \cdot 5$ & 0.3 & $8 \cdot 3$ & 350 \\
\hline Marvel $(10 \mathrm{~g})$ & $7 \cdot 8$ & 0.2 & $5 \cdot 3$ & 228 \\
\hline Nesquik (10 g) & $8 \cdot 0$ & $0 . \overline{3}$ & 0.3 & 152 \\
\hline Rice Krispies (30 g) & 26.9 & 0.3 & $1 \cdot 8$ & 472 \\
\hline Banana $(100 \mathrm{~g})$ & $23 \cdot 2$ & 0.3 & $1 \cdot 2$ & 403 \\
\hline Total & 78.4 & 41.4 & $16 \cdot 9$ & 3083 \\
\hline \multicolumn{5}{|l|}{ Lunch } \\
\hline Cheese pizza $(200 \mathrm{~g})$ & $66 \cdot 2$ & $5 \cdot 4$ & $20 \cdot 0$ & 1670 \\
\hline Lettuce $(30 \mathrm{~g})$ & 0.5 & 0.2 & 0.3 & 18 \\
\hline Cucumber (20 g) & 0.3 & 0 & 0.1 & 8 \\
\hline Tomatoes (45 g) & 1.4 & $0 \cdot 1$ & 0.3 & 33 \\
\hline Total & 68.4 & $5 \cdot 7$ & $20 \cdot 7$ & 1729 \\
\hline
\end{tabular}

*Determined from food tables and manufacturers' data. 
Table 2. Fatty acid composition ( $\mathrm{g} / 100 \mathrm{~g}$ fatty acids) of breakfast ${ }^{\star}$

\begin{tabular}{lcccc}
\hline Mixed-meal enriched with... & $n$-6 PUFA & $n$-3 PUFA & MUFA & SFA \\
\hline SFA & 11 & 19 & 17 & $50 \|$ \\
MUFA & 15 & 20 & $72 \S$ & 40 \\
$n-6$ PUFA & $74 \dagger$ & 39 & 11 & 10 \\
$n-3$ PUFA & 0 & $22 \ddagger$ & 0 & 0
\end{tabular}

PUFA, polyunsaturated fatty acids; MUFA, monounsaturated fatty acids; SFA, saturated fatty acids.

* Determined by GC.

† Comprises $100 \%$ linoleic acid $(18: 2 n-6)$

$\ddagger$ Comprises $50 \%$ eicosapentaenoic acid $(20: 5 n-3)$ and $32 \%$ docosahexaenoic acid

(22:6n-3).

§ Comprises $99 \%$ oleic acid $(18: 1 n-9)$.

|l Comprises $86 \%$ palmitic acid (16:0).

Bucks., UK), exhibiting both inter- and intra-assay CV of less than $10 \%$.

\section{Insulin sensitivity}

$\mathrm{S}_{\mathrm{I}}$ was assessed in the postprandial state using a recently described minimal model index (Caumo et al. 2000). The model provides an estimate of $\mathrm{S}_{\mathrm{I}}$ following carbohydrate ingestion in each individual and following each meal. The $S_{\text {I }}$ calculated by this method utilizes cumulative integrated AUC measures of both insulin and glucose concentration assuming that the total glucose disposal from the system after $240 \mathrm{~min}$ (or when basal values have been reached) equals the glucose entering the peripheral circulation allowing for first-pass extraction by the liver. Insulinindependent mechanisms also contribute to total glucose disposal and a constant rate of glucose effectiveness (GE) has been assumed for the whole time interval.

$$
\mathrm{S}_{\mathrm{I}(\text { oral })}=\mathrm{f} \times \mathrm{D}_{\text {oral }} \frac{\frac{\operatorname{AUC}(\Delta \mathrm{g}(\mathrm{t}) / \mathrm{g}(\mathrm{t}))}{\mathrm{AUC}(\Delta \mathrm{g}(\mathrm{t}))}-\mathrm{GE} \times \operatorname{AUC}(\Delta \mathrm{g}(\mathrm{t}) / \mathrm{g}(\mathrm{t}))}{\operatorname{AUC}(\Delta \mathrm{i}(\mathrm{t}))}
$$

where $\mathrm{D}_{\text {Oral }}$ is the dose of ingested carbohydrate/unit of body weight $(\mathrm{mg} / \mathrm{kg})$ and $\mathrm{f}$ is the fraction of ingested carbohydrate reaching the peripheral circulation as glucose. AUC was calculated from time zero until the end of the test, and GE was fixed at 0.0024 litres $/ \mathrm{kg} \times$ min (Best et al. 1996). The test meals provided in our study contained a significant proportion of starch as the source of carbohydrate, and as the appearance of certain starch-derived glucose fractions into the circulation has been shown to be slower than that of glucose, a nominal value for $\mathrm{f}$ of 0.6 was chosen for all subjects (Robertson et al. 2002b).

\section{Statistical analysis}

Time-course data were analysed by repeated measures ANOVA when normally distributed with post-hoc least significant difference method where appropriate using SPSS (SPSS Inc., Chicago, IL, USA). A level of $P<0.05$ was taken as significant.

\section{Results}

There was no difference in the apparent palatability of the test meals due to the use of a deodorized fish oil. The protocol adopted for the present study proved acceptable to the subjects and all elements of the protocol were completed.

\section{Plasma insulin}

Fig. 1 illustrates the postprandial insulin response following the four high-fat breakfast meals of differing fatty acid composition (at time zero) followed by four identical lunches (at $300 \mathrm{~min}$ ). All subjects exhibited the typical biphasic pattern with concentrations returning to baseline between the two meals. The postprandial response to the first high-fat meal was both of greater magnitude $(P=0.019)$ and earlier $(P=0.004)$ than the second low-fat meal. The response following the breakfast rich in SFA was significantly different from the other three high-fat meals $(P=0 \cdot 006$, repeated measures ANOVA) demonstrated

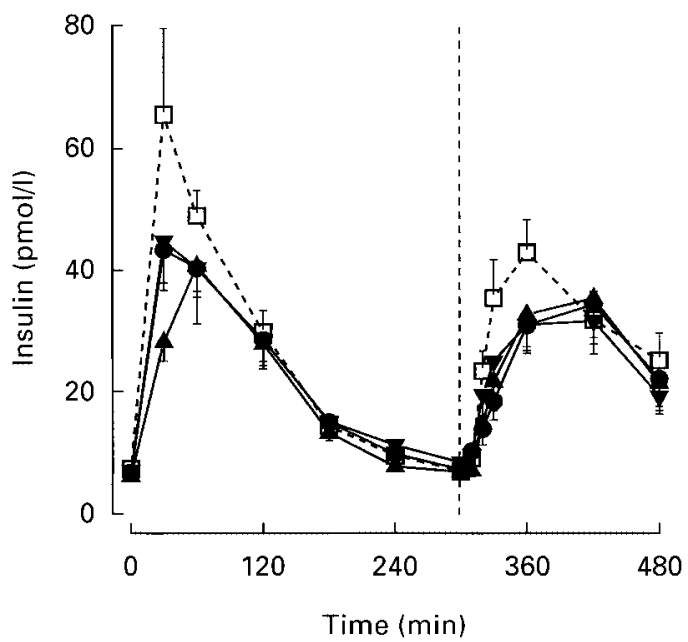

Fig. 1. Plasma insulin concentrations following a high-fat breakfast (at time zero) and a low-fat lunch (at $300 \mathrm{~min},---)$ where the breakfast meal was rich in saturated fatty acids ( $\square$ ), n-6 polyunsaturated fatty acids $(\boldsymbol{\nabla}), n-3$ polyunsaturated fatty acids $(\boldsymbol{\Delta})$ and monounsaturated fatty acids $(\bullet)$. The lunch was identical for all four visits. Mean values for ten women are shown and SEM are represented by vertical bars. Repeated measures ANOVA showed a significant time effect $(P<0.001)$ and a significant meal effect $(P=0.006)$. Post-hoc analysis (least significant difference method) showed that the insulin response following the saturated fatty acid meal was significantly different from the other three meals $(P<0.05)$. 


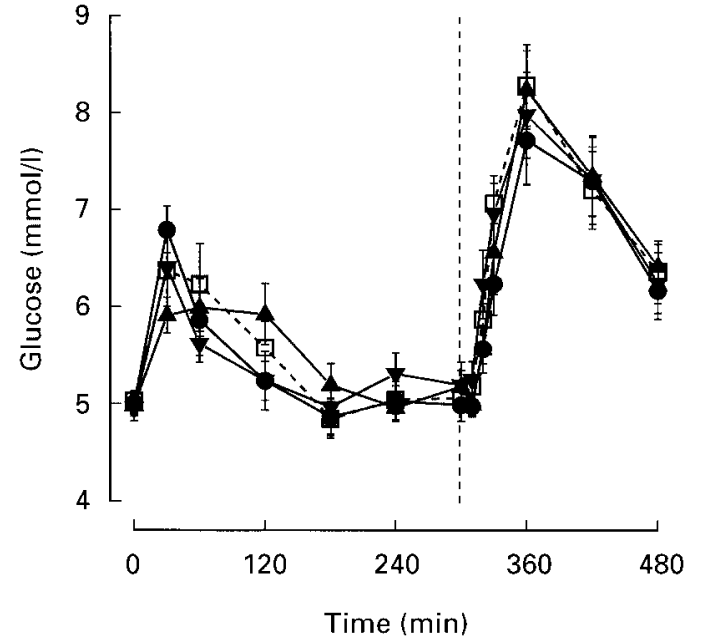

Fig. 2. Plasma glucose concentrations following a high-fat breakfast (at time zero) and a low-fat lunch (at $300 \mathrm{~min},---)$ where the breakfast meal was rich in saturated fatty acids acids $(\square), n-6$ polyunsaturated fatty acids $(\mathbf{\nabla}), n-3$ polyunsaturated fatty acids $(\boldsymbol{\Lambda})$ and monounsaturated fatty acids $(\bullet)$. The lunch was identical in all four studies. Mean values for ten women are shown and SEM are represented by vertical bars. Repeated measures ANOVA showed a significant time effect only $(P<0.001)$.

by higher insulin concentrations after both breakfast and lunch.

\section{Plasma metabolites}

The plasma glucose response following the four high-fat breakfast meals of differing fatty acid composition and the four identical low-fat lunch meals are shown in Fig. 2. All subjects again exhibited a biphasic pattern with glucose concentrations returning to baseline between the two meals. The peak postprandial response following the second low-fat meal (68 g carbohydrate) was significantly greater than that following the first high-fat meal $(78 \mathrm{~g}$ carbohydrate) $(P<0.001)$. The fatty acid composition of the test breakfast had no effect on the postprandial plasma glucose response at breakfast or lunch.

Fig. 3 shows the plasma NEFA response following the four high-fat breakfast meals. NEFA levels were initially suppressed reaching a nadir at 120 min before increasing to reach pre-meal levels again by $300 \mathrm{~min}$. After the second low-fat, high-carbohydrate meal there was a peak in NEFA concentration before levels were again suppressed. Following the breakfast rich in SFA the rise in plasma NEFA following initial suppression was significantly higher $(P=0.003)$ with a higher peak after the lunch meal $30 \mathrm{~min}$ after ingestion.

\section{Insulin sensitivity}

The $\mathrm{S}_{\mathrm{I}}$ following the SFA-rich breakfast was significantly lower than with the other three high-fat meals (SFA, $1.38 ; n$-6 PUFA, $1.87 ; n-3$ PUFA, 1.96 and MUFA, $2 \cdot 13 \times$ $10^{-4}$ litres $/ \mathrm{kg} / \mathrm{min}$ per $\left.\mathrm{mU} / \mathrm{l}, P=0 \cdot 038\right)$. The $\mathrm{S}_{\mathrm{I}}$ following lunch was lower than that following breakfast $(P=0.009)$ irrespective of breakfast fatty acid composition. However,

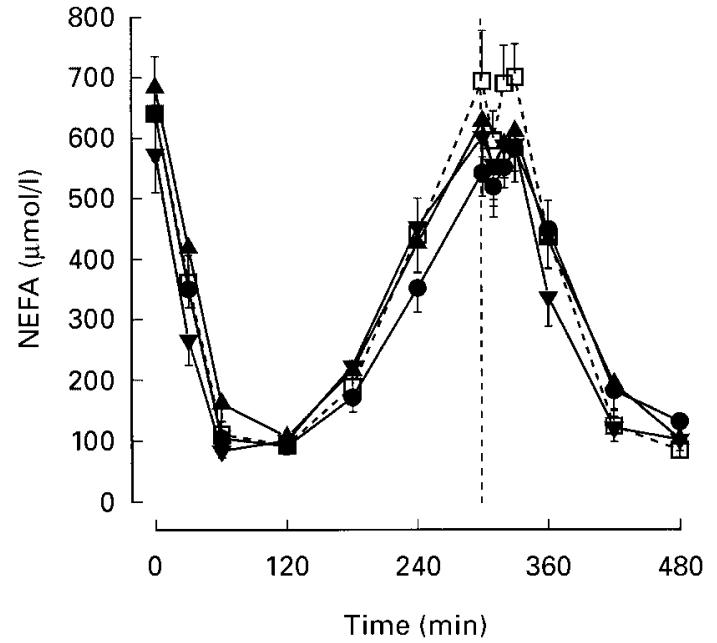

Fig. 3. Plasma non-esterified fatty acid (NEFA) concentrations following a high-fat breakfast (at time zero) and a sequential low-fat lunch (at $300 \mathrm{~min},--)$ where the breakfast meal was rich in saturated fatty acids acids $(\square), n-6$ polyunsaturated fatty acids $(\boldsymbol{\nabla}), n-3$ polyunsaturated fatty acids $(\boldsymbol{\Lambda})$ and monounsaturated fatty acids $(\bullet)$. The lunch was identical in all four studies. Mean values for ten women are shown and SEM are represented by vertical bars. Repeated measures ANOVA showed a significant effect of time $(P<0.001)$ and a significant time $v$. meal interaction $(P=0.003)$.

the $S_{I}$ for the four breakfast test meals exhibited the same rank order after both breakfast and lunch (Fig. 4).

\section{Discussion}

In the present study we have demonstrated that the acute ingestion of a mixed meal rich in SFA results in significantly elevated postprandial insulin levels compared with meals enriched with other dietary fats (MUFA, $n-6$ PUFA or $n$-3 PUFA). Utilizing a minimal model approach (Caumo et al. 2000), the palm-oil meal resulted in a lower

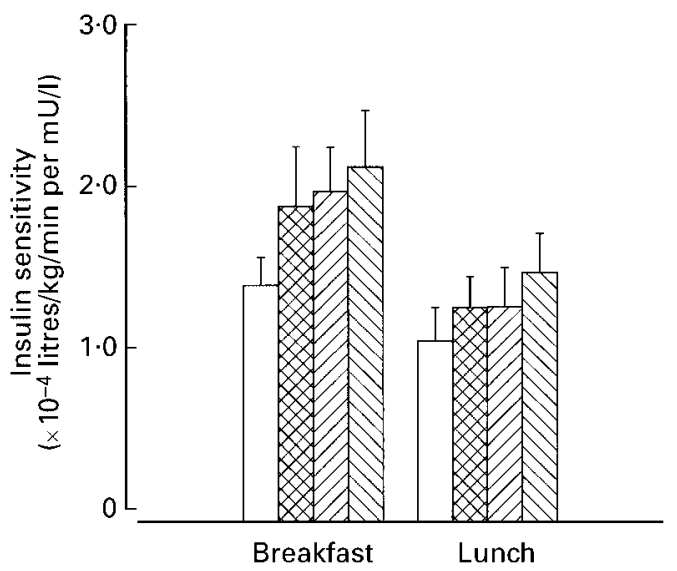

Fig. 4. Oral insulin sensitivity following a high-fat breakfast and a sequential low-fat lunch where the breakfast meal was rich in saturated fatty acids acids ( $\square$ ), n-6 polyunsaturated fatty acids (网), n-3

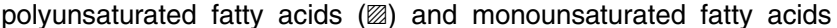
$(\mathbb{Q})$. Mean values for ten women are shown and SEM are represented by vertical bars. Repeated measures ANOVA showed a significant effect between breakfast and lunch $(P=0.009)$ and a significant difference between the breakfast fatty acid composition ( $P=0.038$ ). 
postprandial $S_{\text {I }}$ after breakfast. It is accepted that a fatty breakfast has deleterious effects on glucose tolerance and $S_{I}$ to a lunch-time meal, presumably through effects on NEFA concentrations (Frape et al. 1998). In the present study we were able to clarify the effects of breakfast fatty acid composition on these parameters. Although insulin levels and $S_{I}$ were reduced at lunch-time compared with breakfast irrespective of breakfast fatty acid composition, the $S_{I}$ following lunch showed the same rank order as after breakfast, despite the lunch-time meal being identical in all cases (Fig. 4).

The effects of meal fatty acid composition on acute glucose-stimulated insulin levels following a single meal have been demonstrated previously. Gannon et al. (1993) found that adding butter (high in SFA) to a potato meal gave a higher insulin and C-peptide response than when the potato was given alone. However, when Welch et al. (1987) added maize oil (high in PUFA) to a potato meal they found the insulin response to be reduced. Comparison between such mixed-meal studies is difficult due to differences in test meal composition, in particular variations in the amount of fat, fatty acid enrichment and type of carbohydrate (Gatti et al. 1992). It might be supposed that variations in the secretion of gut hormones (glucagon-like peptide 1, cholecystokinin and glucose insulinotropic polypeptide) could potentiate differential insulin secretion according to fatty acid composition (Zampelas et al. 1994). Whilst it is true that stimulation of these hormones by fat-containing meals could explain the enhanced insulin response compared with when fat is absent from a meal, gut hormone concentrations measured during the present study show that palm oil is not a more potent secretagogue than the other dietary oils (Robertson et al. 2002a). Other possibilities include variable effects of meal fatty acid composition on circulating plasma NEFA, which have been shown to be stimulatory towards the pancreatic $\beta$-cell (Greenough et al. 1967). Short-term (3 h) elevation of NEFA in rats (Grill \& Qvigstad, 2000) and human subjects (Beysen et al. 2002) increases glucose-stimulated insulin secretion although different fatty acids may not be equal in their insulinotropic potency. In this respect the results from the literature are contradictory. In vitro studies have clearly shown that SFA are the most potent stimulators of insulin secretion in isolated rat islets (Stein et al. 1997), whereas in human plasma there is some evidence that MUFA dominate in terms of insulinotropic potential (Beysen et al. 2002).

In the plasma, dietary chylomicron-triacylglycerol is acted upon by lipoprotein lipase to release NEFA and up to $50 \%$ of these liberated NEFA escape 'capture' by tissues and enter the plasma NEFA pool directly (Coppack et al. 1992). Although specific fatty acid measurements were not made in the present study, similar studies using high-fat, high-carbohydrate test meals have shown postprandial changes in the plasma NEFA profile to resemble the fatty acid composition of the test meal (Fielding et al. 1996). Therefore in the postprandial period following each of our high-fat breakfasts, we suggest that due to the action of insulin in suppressing hormone-sensitive lipase, the majority of the NEFA pool would be of dietary origin. A high saturated fatty acids:unsaturated fatty acids ratio in the NEFA pool following the palm-oil breakfast may therefore have promoted hypersecretion of insulin (Stein et al. 1997). This is contrary to other work by our group (Beysen et al. 2002), in which MUFA were found to promote greater insulin secretion than SFA. However, in that study NEFA levels were artificially elevated with an infusion of heparin. In the present study the absolute concentration of plasma NEFA fell in the postprandial period and so whether the composition of the NEFA pool is as important as the combined concentration of all circulating NEFA remains to be determined.

The ability to calculate $S_{I}$ from glucose and insulin measurements following mixed meals adds a new dimension to postprandial experiments (Caumo et al. 2000). In this setting the minimal model approach has distinct advantages over the classic hyperglycaemic-hyperinsulinaemic clamp methods as it allows gastrointestinal factors (gastric emptying and gastrointestinal hormone release) to be accounted for in the physiological non-steady state. However, the value obtained from the minimal model cannot be readily compared with those obtained from clamp experiments. The true relationship between the plasma glucose and insulin levels may be masked by the secondary effects of hormones such as glucagon-like peptide 1 on concentrations of insulin, glucagon, glucose and NEFA levels postprandially.

In the acute situation there is reported to be a 3-4h delay between the rise in plasma NEFA and the inhibition of insulin-stimulated glucose uptake (Boden et al. 2001), making a direct effect of NEFA on insulin action in the present study unlikely. Another possible explanation could be a reduction in muscle $S_{I}$ due to the accumulation of intramyocellular fat within muscle tissue, although whether this is relevant in the acute postprandial period remains to be established.

In conclusion, ingestion of a single meal rich in SFA led to an elevated insulin concentration with a reduction in $S_{I}$ when compared with other dietary fats with follow-on effects for a subsequent meal.

\section{Acknowledgements}

The authors thank David Araujo-Vilar from Servicio de Endocrinoloxia e Nutricion, Hospital Xeral, Spain for use of the computer program for calculation of $\mathrm{S}_{\mathrm{I}(\text { Oral) }}$.

\section{References}

Best JD, Kahn SE, Ader M, Watanabe RM, Ni TC \& Bergman RN (1996) Role of glucose effectiveness in the determination of glucose tolerance. Diabetes Care 19, 1018-1030.

Beysen C, Karpe F, Fielding BA, Clark A, Levy JC \& Frayn KN (2002) Interaction between specific fatty acids, GLP-1 and insulin secretion in humans. Diabetologia (In the Press).

Boden G, Lebed B, Schatz M, Homko C \& Lemieux S (2001) Effects of acute changes of plasma free fatty acids on intramyocellular fat content and insulin resistance in healthy subjects. Diabetes 50, 1612-1617.

Caumo A, Bergman RN \& Cobelli C (2000) Insulin sensitivity from meal tolerance tests in normal subjects: a minimal 
model index. Journal of Clinical Endocrinology and Metabolism 85, 4396-4402.

Coppack SW, Evans RD, Fisher RM, Frayn KN, Gibbons GF, Humphreys SM, Kirk MJ, Potts JL \& Hockaday TDR (1992) Adipose tissue metabolism in obesity: lipase action in vivo before and after a mixed meal. Metabolism 41, 264-272.

Ercan N, Nuttall FQ \& Gannon MC (1994) Effect of added fat on the plasma glucose and insulin response to ingested potato given in various combinations as two meals in normal individuals. Diabetes Care 17, 1453-1459.

Evans K, Kuusela PJ, Cruz ML, Wilhelmova I, Fielding BA \& Frayn KN (1998) Rapid chylomicron appearance following sequential meals: Effects of second meal composition. British Journal of Nutrition 79, 425-429.

Fielding BA, Callow J, Owen RM, Samra JS, Matthews DR \& Frayn KN (1996) Postprandial lipaemia: The origin of an early peak studied by specific fatty acid intake during sequential meals. American Journal of Clinical Nutrition 63, 36-41.

Frape DL, Williams NR, Rajput Williams J, Maitland BW, Scriven AJ, Palmer CR \& Fletcher RJ (1998) Effect of breakfast fat content on glucose tolerance and risk factors of atherosclerosis and thrombosis. British Journal of Nutrition 80, 323-331.

Frape DL, Williams NR, Scriven AJ, Palmer CR, O'Sullovan K \& Fletcher RJ (1997a) Diurnal trends in responses of blood plasma concentrations of glucose, insulin and C-peptide following high- and low-fat meals and their relation to fat metabolism in healthy middle-aged volunteers. British Journal of Nutrition 77, 523-535.

Frape DL, Williams NR, Scriven AJ, Palmer CR, O'Sullovan K \& Fletcher RJ (1997b) Effects of high- and low-fat meals on the diurnal response of plasma lipid metabolite concentrations in healthy middle-aged volunteers. British Journal of Nutrition 77, 375-390.

Gannon MC, Nuttall FQ, Westphal SA \& Seaquist ER (1993) The effect of fat and carbohydrate on plasma glucose, insulin, c-peptide, and triglycerides in normal male subjects. Journal of the American College of Nutrition 12, 36-41.

Gatti E, Noe D, Pazzucconi F, Gianfranceschi G, Porrini M, Testolin G \& Sirtori CR (1992) Differential effect of unsaturated oils and butter on blood glucose and insulin response to carbohydrate in normal volunteers. European Journal of Clinical Nutrition 46, 161-166.

Greenough WB, Crespin SR \& Steinberg D (1967) Hypoglycemia and hyperinsulinaemia response to raised free-fatty acid levels. Lancet ii, 1334-1336.

Grill V \& Qvigstad E (2000) Fatty acids and insulin secretion. British Journal of Nutrition 83, Suppl. 1, S79-S84.

Jackson KG, Robertson MD, Fielding BA, Frayn KN \& Williams CM (2002) Olive oil increases the number of triacylglycerol-rich chylomicron particles compared with other oils: an effect retained when a second standard meal is fed. American Journal of Clinical Nutrition (In the Press).

Joannic J-L, Auboiron S, Raison J, Basdevant A, Bornet F \& Guy-Grand B (1997) How the degree of unsaturation of dietary fatty acids influences the glucose and insulin responses to different carbohydrates in mixed meals. American Journal of Clinical Nutrition 65, 1427-1433.

Pedersen A, Marckmann P \& Sandstrom B (1999) Postprandial lipoprotein, glucose and insulin responses after two consecutive meals containing rapeseed oil, sunflower or palm oil with or without glucose at the first meal. British Journal of Nutrition 82, 97-104.

Rasmussen O, Lauszus FF, Christiansen C, Thomsen C \& Hermansen K (1996) Differential effects of saturated and monounsaturated fat on blood glucose and insulin responses in subjects with non-insulin dependent diabetes mellitus. American Journal of Clinical Nutrition 63, 249-253.

Reaven GM (1988) Role of insulin resistance in human disease. Diabetes 31, 670-673.

Robertson MD, Jackson KG, Fielding BA, Williams CM \& Frayn $\mathrm{KN}$ (2002a) Acute ingestion of triacylglycerol rich in $\mathrm{n}-3$ polyunsaturated fatty acids results in rapid gastric emptying. American Journal of Clinical Nutrition 76, 232-238.

Robertson MD, Livesey G \& Mathers JC (2002b) Quantitative kinetics of glucose uptake and disposal following a ${ }^{13} \mathrm{C}$ labelled starch-rich meal: comparison of male and female subjects. British Journal of Nutrition 87, 569-577.

Stein DT, Stevenson BE, Chester MW, Basit M, Daniels M, Turley SD \& McGarry JD (1997) The insulinotropic potency of fatty acids is influenced profoundly by their chain length and degree of saturation. Journal of Clinical Investigation 100, 398-403.

Thomsen C, Rasmussen O, Lousen T, Holst JJ, Fenselau S, Schrezenmeir J \& Hermansen K (1999) Differential effects of saturated and monounsaturated fatty acids on postprandial lipemia and incretin responses in healthy subjects. American Journal of Clinical Nutrition 69, 1135-1143.

Vessby B (2000) Dietary fat and insulin action in humans. British Journal of Nutrition 83, Suppl. 1, S91-S96.

Welch IM, Bruce C, Hill SE \& Read NW (1987) Duodenal and ileal lipid suppresses postprandial blood glucose and insulin responses in man: possible implications for the dietary management of diabetes mellitus. Clinical Science 72, 209-216.

Zampelas A, Murphy M, Morgan LM \& Williams CM (1994) Postprandial lipoprotein lipase, insulin and gastric inhibitory polypeptide responses to test meals of different fatty acid composition: Comparison of saturated, n- 6 and n-3 polyunsaturated fatty acids. European Journal of Clinical Nutrition 48, $849-858$ 